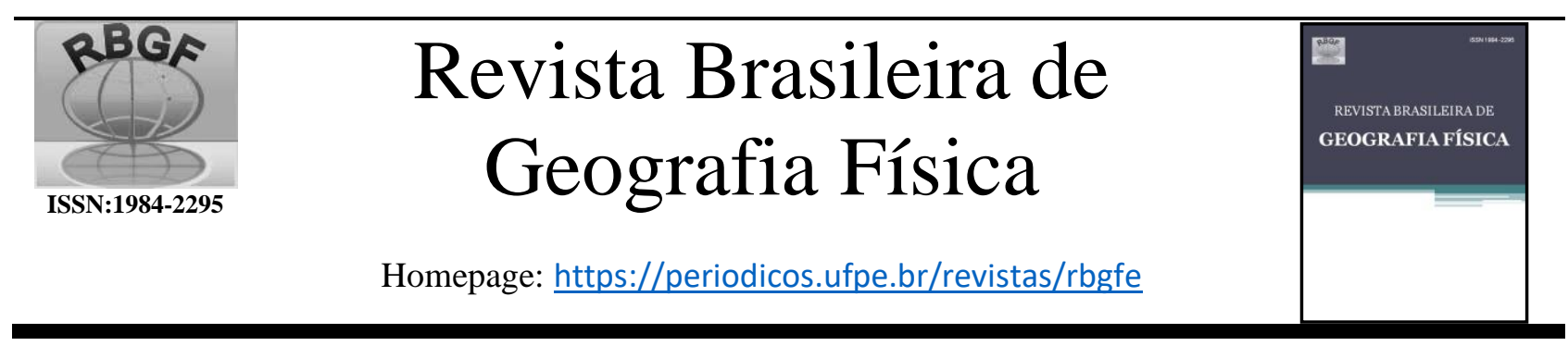

\title{
Validação de mapa de vulnerabilidade a erosão por aprendizagem de máquina
}

\author{
Renata Cristina Mafra ${ }^{1}$; Mayara Maezano Faita Pinheiro ${ }^{2}$; Rejane Ennes Cicerelli ${ }^{3}$; Lucas Prado \\ $\mathrm{Osco}^{4}$; Marcelo Rodrigo Alves ${ }^{5}$; Ana Paula Marques Ramos ${ }^{6}$

\footnotetext{
${ }^{1,2}$ Universidade do Oeste Paulista, Programa de Pós-Graduação em Meio Ambiente e Desenvolvimento Regional. Rodovia Raposo Tavares, km 572, 19067-175, Presidente Prudente, SP, Brasil. *renatacmafra@outlook.com.

${ }^{3}$ Universidade de Brasília, Instituto de Geociências. Asa Norte, 70910-900 Brasília - DF, Brazil.

${ }^{4}$ Universidade Federal do Mato Grosso do Sul, Pós-doutorando no Programa de Recursos Naturais. Cidade Universitária, 79070-900, Campo Grande, MS, Brasil.

5,6 Universidade do Oeste Paulista, Docente do Programa de Pós-Graduação em Meio Ambiente e Desenvolvimento Regional. Rodovia Raposo Tavares, km 572, 19067-175, Presidente Prudente, SP, Brasil.
}

Artigo recebido em 23/10/2019 e aceito em 22/03/2020

\section{R E S U M O}

$\mathrm{O}$ processo erosivo é um fenômeno que acontece devido às condições climáticas ou uso inadequado da terra. $\mathrm{O}$ mapeamento dos níveis de vulnerabilidade à erosão de uma área pode ocorrer usando diferentes modelos de inferência geográfica. No entanto, definir o método apropriado é ainda uma questão a ser respondida. Este trabalho apresenta uma abordagem de validação de mapa de vulnerabilidade à erosão elaborado por diferentes métodos de inferência. Como estudo de caso, adotou-se uma bacia hidrográfica e considerou-se os seguintes critérios: geomorfologia, pedologia, declividade, densidade de drenagem e cobertura da terra. Dentre os métodos testados tem-se: Combinação Linear Ponderada (CLP) e três operadores Fuzzy: soma algébrica, produto algébrico e gamma, variando o expoente " $\gamma$ " entre os valores 0,$4 ; 0,6$ e 0,8. Os pesos dos critérios foram definidos com base no Processo Analítico Hierárquico. A validação dos mapas ocorreu usando 1902 pontos, sendo 951 pontos de erosão na área, definidos com base em imagens do Google Earth Pro, e 951 pontos sem erosão, gerados aleatoriamente no QGIS 3.8. O modelo de regressão logística foi usado parar comparar o desempenho de cada mapa ao apontar as áreas com maior e menor grau de vulnerabilidade. A melhor modelagem foi alcançada com o operador Fuzzy gamma quando parametrizado com $\gamma=0,6$. Embora o CLP seja a abordagem recorrente em estudos ambientais envolvendo inferência geográfica, nossos resultados demostram que outros operadores podem produzir resultados mais próximos aos encontrados com a realidade observada em campo. Palavras-chave: Inferência geográfica; análise multicritério; validação de dados; impacto ambiental.

\section{Machine learning erosion and vulnerability map validation}

\section{A B S T R A C T}

Erosion is a natural phenomenon that happens in all ecosystems, whether due to weather conditions or inappropriate land use. Mapping the erosion vulnerability levels of an area can occur using different methods of geographic inference. However, defining the appropriate method is still a question to be answered. This paper presents an erosion vulnerability map validation approach elaborated by different inference methods. As a case study, a watershed was adopted and the following criteria were considered: geomorphology, pedology, slope, drainage density and land cover. Among the tested methods are: Weighted Linear Combination (WLC) and three Fuzzy operators: algebraic sum, algebraic product and gamma, varying the exponent " $\gamma$ " between the values $0.4 ; 0.6$ and 0.8 . The weights of the criteria were defined based on the Hierarchical Analytical Process. The validation of the maps took place using 1902 points, with 951 erosion points in the area defined based on Google Earth Pro images and 951 points without erosion randomly generated in QGIS 3.8. The logistic regression model was used to compare the performance of each map by pointing out the areas with the highest and lowest degree of vulnerability. The best modeling was achieved with the Fuzzy gamma operator when parameterized with $\gamma=0.6$. Although WLC is the recurring approach in environmental studies involving geographic inference, our results show that other operators can produce results closer to those encountered with the reality observed in the field. Keywords: Geographical inference; multicriteria analysis; data validation; environmental impact. 


\section{Introdução}

A erosão é um fenômeno de superfície que ocorre pelo processo físico de desintegração, transporte e deposição de partículas do solo causadas pela água da chuva e escoamento (Silva et al., 2012; Jesuz et al., 2019). No Brasil, o risco de degradação do solo é elevado devido às condições climáticas tropicais dominantes. Além disso, o uso inadequado da terra, como substituições de cobertura vegetal por outros tipos de cobertura em áreas frágeis, pode promover o surgimento de feições erosivas (Ding et al., 2013, Porto et al.; 2019).

Uma vez que ocorre a erosão no solo, os sedimentos podem ser carregados pelo escoamento superficial até os corpos hídricos e, dependendo da sua concentração, pode comprometer a qualidade da água (Meneses et al., 2015; Shi et al., 2017). As áreas vulneráveis à erosão podem apresentar influências de fatores naturais (declividade, cobertura vegetal, tipo do solo) ou de fatores antrópicos (uso do solo) (Silva et al., 2014; Reis et al., 2017; Silva et al., 2017). Assim, entender o grau de vulnerabilidade à erosão de uma área é requisito necessário para realizar um planejamento ambiental eficiente e evitar desastres ambientais (Nascimento et al, 2016; Ribeiro et al. 2016).

As geotecnologias possibilitam identificar e estabelecer um controle e/ou direcionamento das atividades antrópicas de uso da terra e também otimiza a produção de diversos produtos cartográficos, como um mapa de vulnerabilidade, que são importantes para o diagnóstico ambiental de uma área (Bianchini et al., 2019). Um mapa de vulnerabilidade é oriundo da combinação de várias informações geográficas, caracterizando um processo de inferência geográfica com análise multicritério. Esse tipo de análise envolve um conjunto de alternativas de decisão, as quais são baseadas em vários critérios de avaliação (Malczewski, 2010). No contexto de problemas espaciais, esses critérios são combinados em um processo cartográfico denominado de álgebra de mapas, resultando em um mapa síntese. Os Sistemas de Informação Geográfica (SIG) são um tipo de geotecnologias que otimizam a resolução de problemas espaciais de decisão do tipo multicritério, pois permitem integrar de forma rápida e eficaz dados geográficos de diferentes fontes e formatos (Longley et al. 2013).

Diante de uma tomada de decisão que envolve a consideração de multicritérios, uma etapa crucial é a definição da contribuição relativa de cada critério. Uma das técnicas mais tradicionais para desenvolver a análise multicritério é a proposta por Thomas Saaty em 1978 denominada de Processo Analítico Hierárquico (AHP - Analytic Hierarchy Process) (Saaty, 1980). O princípio é atribuir o peso aos critérios a partir de uma comparação pareada entre esses. Para tanto, Saaty propôs uma escala de 1 a 9, denominada de Escala Fundamental, sendo o valor 1 atribuído aos critérios de mesma importância relativa, e o valor 9 aos critérios de importância absoluta em relação aos demais (Saaty, 1980).

A partir da definição dos pesos dos critérios pelo AHP, gera-se o mapa síntese pela Combinação Linear Ponderada desses critérios ou, menos tradicionalmente, pela Média Ponderada Ordenada dos critérios (Valente \& Vettorazzi, 2005; 2008). A análise multicriterial usando o AHP é empregada em diferentes áreas atendendo a distintas aplicações, incluindo o diagnóstico ambiental de bacias hidrográficas, como em Ross (1994), Fushimi et al. (2013), Nossack et al. (2014) Valente \& Vettorazzi (2008, 2016); Gonçalves et al. (2016), Mello et al. (2018), Rodrigues et al. (2018) e Fushimi et al. (2018).

Outro importante método no processo de inferência geográfica é a lógica Fuzzy, que corresponde a uma extensão da lógica Booleana visando manipular o conceito de "verdade parcial" (Mota et al., 2019), isto é, valores compreendidos entre "completamente verdadeiro" e "completamente falso" (Câmara et al., 2001). A lógica Fuzzy pode ser aplicada usando diferentes operadores para a combinação dos critérios na análise multicriterial, tais como operador de soma algébrica, operador produto algébrico e o operador gamma. Utilizar conjuntos Fuzzy tornam os conceitos imprecisos, encontrados no meio físico, mais próximos da realidade (Gonçalves et al. 2016).

Embora o uso do processo de inferência geográfica seja considerado um clássico para a resolução de problemas de decisão uni e multicritério, e que existam diferentes modelos lógicos que podem ser empregados (por exemplo, AHP, como a Combinação Linear Ponderada, ou a lógica Booleana, lógica Fuzzy), ainda não há um consenso de como a acurácia dos mapas sínteses deve ser validada. Há trabalhos que nem mesmo realizam um controle de qualidade dos produtos gerados por esses modelos, e outros que validam com observações de campo (Samizava et al., 2008; Fushimi et al., 2013; Fushimi et al., 2018; Trevisan et al., 2018). Todavia, nota-se que, em geral, há o uso de poucas amostras de campo, como no trabalho de Samizava et al. (2008) que adotam cinco pontos de validação para controle de qualidade do mapa síntese. Isso levanta o questionamento sobre se esse número reduzido de amostras é representativo para toda a área estudada. 
Recentemente, as técnicas de inteligência artificial, mais particularmente, os algoritmos de Aprendizagem de Máquina vêm sendo aplicadas na resolução de problemas cartográficos. Dentre esses vários algoritmos, a regressão logística é uma abordagem adequada para situações nas quais se deseja predizer a presença ou ausência de uma determinada característica (presença ou ausência de erosão, por exemplo), baseado em valores de um conjunto de variáveis independentes. No entanto, até o presente momento, não se sabe do uso de algoritmos de aprendizagem de máquina para a validação de mapas obtidos no processo de inferência geográfica.

Nesse sentido, esse trabalho apresenta uma proposta de validação da acurácia de mapa de vulnerabilidade à erosão utilizando um denso conjunto amostral processado pelo algoritmo regressão logística em ambiente de aprendizagem de máquina. Para esta finalidade, elaboramos o mapa de vulnerabilidade à erosão aplicando diferentes modelos lógicos de inferência geográfica, como Combinação Linear Ponderada, sendo os pesos dos critérios determinado pelo método AHP, e lógica Fuzzy com os operadores algébrico soma e produto, e o operador gamma. Com isso, esse trabalho traz não somente uma abordagem generalizada de como validar a acurácia de mapa síntese a partir de técnicas de inteligência artificial, como também apresenta achados relevantes sobre o nível de vulnerabilidade à erosão que se encontra a bacia de estudo de caso, reforçando a necessidade de mitigação dos efeitos antrópicos sobre esta.

\section{Material e métodos}

Área de Estudo

A bacia hidrográfica do rio Pirapozinho (Figura 1) foi adotada como área estudo de caso. Com uma área de aproximadamente $1.000 \mathrm{~km}^{2}$, esta bacia está inserida na $22^{\text {a }}$ Unidade de Gerenciamento de Recursos Hídricos do Pontal do Paranapanema (UGRHI-22). Essa bacia possui diferenciações físicas quando comparada as demais bacias da UGRHI-22 (Ramos et al., 2018), indicando diferentes cenários de vulnerabilidade à erosão. Outra característica importante da bacia do rio Pirapozinho, e que a torna uma importante área de estudo de caso, é a possibilidade de uso futuro para abastecimento público de água dos núcleos populacionais dentro ou próximos a ela. Ainda na Figura 1 é apresentado a distribuição espacial do conjunto de pontos de validação adotados nesse trabalho.

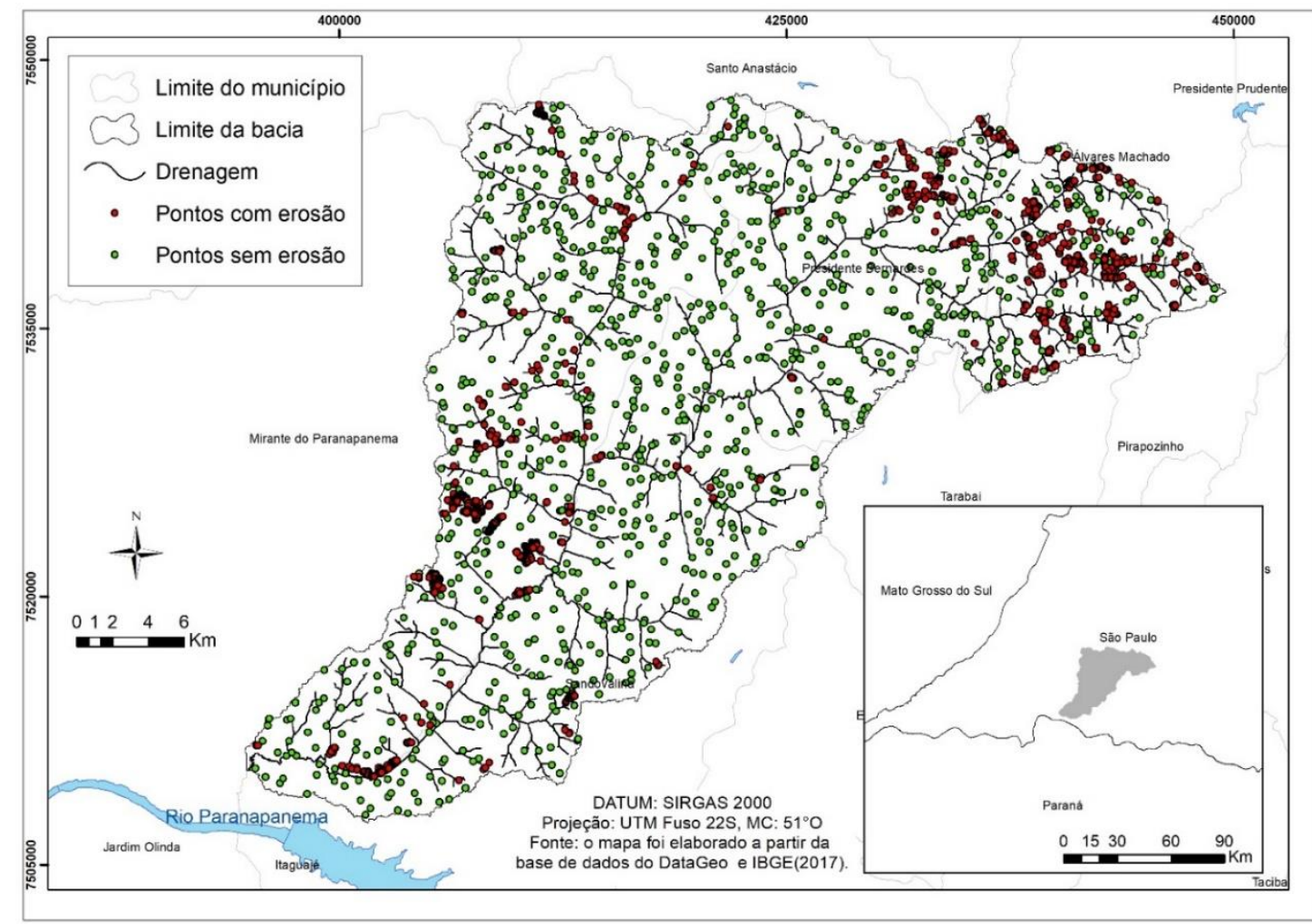

Figura 1. Área de estudo e distribuição espacial dos pontos de validação. 
Organização dos dados cartográficos

Os critérios (variáveis) para a elaboração do mapa de vulnerabilidade à erosão da bacia em estudo foram definidos de acordo com a literatura (Fushimi, 2013; Fushimi, 2018). Tais critérios foram: geomorfologia, pedologia, declividade, densidade de drenagem e uso e cobertura da terra.

a) Geomorfologia: está relacionada com a forma das vertentes e o grau de declividade (Viel et al., 2017), intensificando ou não o grau de transporte de sedimentos;

b) Pedologia: os processos de erosão são acelerados pelo uso inadequado do solo, mudanças climáticas, e por suas características naturais. Os tipos de solo são classificados em diferentes classes por suas propriedades, como teor de matéria orgânica, textura, densidade, porosidade e sua estrutura, que interferem direta ou indiretamente na quebra de agregados e, consequentemente, em seu transporte (Oliveira, 2009);

c) Declividade: quanto maior o nível de declividade, maior é o escoamento superficial e mais suscetível ao processo de erosão está o solo. Portanto, um declive acentuado reduz a taxa de infiltração de água no solo e aumenta a sedimentação da rede de drenagem (Silva et al., 2014).

d) Densidade de drenagem: resulta de uma rede de drenagem e calcula a densidade da rede de drenagem dentro de uma área específica (Dobos et al., 2000), ou seja, identifica onde está o maior fluxo e é uma boa indicação do grau de desenvolvimento de um sistema de drenagem;

e) Uso e cobertura da terra: a substituição de áreas florestais por áreas de usos urbanos e/ou agrícolas pode promover, por exemplo, a redução da qualidade da água devido às erosões causadas pela ausência da vegetação, aumentando o carregamento de sedimentos até os rios (Yang et al., 2016).

Para cada um desses critérios, compilou-se os dados cartográficos existentes para a área de estudo, organizando-os em um banco de dados geográficos. Adotamos o sistema de referência SIRGAS 2000 e a projeção Universal Transverso de Mercator, fuso 22 sul.

A delimitação da área da bacia do rio Pirapozinho foi realizada a partir de processos automáticos (ferramenta Hidrology) no SIG ArcGIS 10.2, usando um Modelo Digital de Superfície, de $30 \mathrm{~m}$ de resolução espacial, coletado na missão SRTM (Shuttle Radar Topograpy Mission) da NASA em 2000. O limite da área da bacia foi utilizado como máscara de recorte para os dados cartográficos de interesse.
Para os critérios 'geomorfologia' e 'pedologia' foram utilizados os dados do Instituto de Pesquisa Tecnológica (IPT), de 1999, na escala 1:250.000. Para critério 'uso e cobertura da terra' os dados foram baixados na base de dados contínua do Instituto Brasileiro de Geografia e Estatística (IBGE) de 2017, escala 1:100.000.

Os dados relacionados aos critérios 'densidade de drenagem' e 'declividade' foram extraídos do MDS SRTM. Para a densidade de drenagem, os processamentos foram divididos em 3 etapas, sendo: 1) preenchimento de depressões no MDS (fill); 2) cálculo da direção do fluxo da água (flow direction) e; geração do fluxo acumulado de água (flow accumulation).

Depois de calculado o fluxo acumulado, criou-se a rede de drenagem considerando apenas as células com um fluxo acumulado maior que 100, representado pelo número 1. Esse valor foi considerado pois atendeu as características da área de estudo, podendo ser usado para outras características por meio de tentativa e erro. A equação (1) a seguir foi utilizada para gerar a rede de drenagem.

$$
\text { Drenagem }=\mathrm{CON}(\text { flowacc }>100,1,0)
$$

O mapa de densidade de drenagem foi obtido pela soma de cada célula do mapa de drenagem em um raio de $1 \mathrm{~km}$, dividido pela área de $1 \mathrm{~km}^{2}$, com a ferramenta focal statistics e Neighborhood CIRCLE no ArcGis 10.2. Assim, obteve-se a quantidade de cursos d'água existentes em um raio de $1 \mathrm{~km}$ na bacia. Esse valor de raio foi definido a partir da inspeção visual da distribuição da drenagem na área da bacia.

\section{Atribuição dos pesos usando o AHP}

$\mathrm{O}$ peso dos critérios reflete a importância desses no processo de tomada de decisão, neste caso, na caracterização do nível de vulnerabilidade à erosão da bacia do rio Pirapozinho. Para expressar a importância, ou a ordem de importância dos critérios definidos nesse estudo de caso, empregou-se o AHP tanto para a definição dos pesos de cada variável (Tabela 1) quanto de suas subclasses (Tabela 2). É importante ressaltar que o peso dos critérios (Tabela 2) foi aplicado somente na inferência pela Combinação Linear Ponderada.

Para cada aplicação do AHP, calculou-se a Razão de Consistência (RC) (Equação 2) considerando a matriz de importância relativa dos critérios.

A RC indica a probabilidade de o peso do critério ter sido gerado aleatoriamente, isto é, se os pesos atribuídos aos critérios são consistentes. 
Tabela 1 - Matriz de pesos das subclasses dos critérios (variáveis) definidos pelo AHP.

\begin{tabular}{lcc}
\hline $\begin{array}{c}\text { Critérios } \\
\text { (classe) }\end{array}$ & Subclasses & Pesos \\
\hline \multirow{3}{*}{ Geomorfologia } & $\begin{array}{c}\text { Colinas Médias } \\
\text { Colinas }\end{array}$ & 0,67521 \\
& Amplas & 0,25314 \\
& Planícies & 0,07265 \\
\hline & Neossolo & 0,59144 \\
Pedologia & Nitossolo & 0,22528 \\
& Argissolo & 0,13101 \\
& Latossolo & 0,05226 \\
\hline & Urbano & 0,47261 \\
Cobertura da & Pecuária & 0,24167 \\
Terra & Agricultura & 0,16384 \\
& Savana & 0,05329 \\
& Floresta & 0,03430 \\
& Floresta & 0,03430 \\
\hline
\end{tabular}

Tabela 2 - Matriz de pesos dos critérios definidos pelo AHP.

\begin{tabular}{lcccccc}
\hline \multicolumn{1}{c}{ Critérios } & Pedologia & Geomorfologia & Vegetação & Declividade & $\begin{array}{c}\text { Densidade } \\
\text { de } \\
\text { drenagem }\end{array}$ & Pesos \\
\hline Pedologia & 1 & & & & & 0,103775 \\
\hline Geomorfologia & 2 & 1 & & & 0,103264 \\
\hline Vegetação & 2 & 2 & 1 & & 0,130656 \\
\hline Declividade & 2 & 4 & 4 & 1 & 1 & 0,399724 \\
\hline $\begin{array}{l}\text { Densidade de } \\
\text { drenagem }\end{array}$ & 2 & 4 & 4 & $1 / 3$ & 0,262581 \\
\hline
\end{tabular}

$\mathrm{O}$ valor do RC deve ser inferior a 0,10 ; caso contrário, a matriz de importância relativa deve ser revista para alterar os valores da comparação pareada (Saaty, 1980)

$$
\mathrm{RC}=\mathrm{IC} / \mathrm{IR}
$$

Em que: IC é o índice de consistência; e IR é o índice aleatório. O IR é um valor tabelado, cujo valor depende da dimensão da matriz (n). O IC é determinado pela razão entre $\left(\lambda_{\max }-\mathrm{n}\right) /(\mathrm{n}-1)$, em que $\lambda_{\max }$ é o autovalor máximo da matriz.

\section{Inferência geográfica por diferentes modelos lógicos}

Antes de realizar a álgebra de mapas, isto é, a combinação dos critérios para a produção do mapa síntese, deve-se padronizar os dados em uma escala comum de valores. Essa padronização foi realizada considerando a escala de 0 e 1 , em que o valor 0 representa as áreas não vulneráveis à erosão, e o valor 1 as áreas que estão totalmente vulneráveis à erosão. Realizamos essa operação por meio da ferramenta Fuzzy Membership do software ArcGIS 10.2. O.

$\mathrm{O}$ mapa de vulnerabilidade à erosão foi elaborado por meio de diferentes modelos lógicos de inferência geográfica, sendo os quais a Combinação Linear Ponderada (CLP) juntamente com o método AHP, e lógica Fuzzy, considerando três operadores distintos: soma algébrica; produto algébrico e; operador gamma.

A Combinação Linear Ponderada, proposta por Voogd (1983), constitui-se da soma dos critérios multiplicados por seus respectivos pesos. Assim como os operadores Fuzzy, a abordagem da CLP é realizar uma operação pixel a pixel (dos mapas dos fatores, ou denominados de critérios), permitindo utilizar critérios com variação contínua. Seu resultado é um mapa quantitativo expressando o grau de importância relativa por meio dos valores numéricos de saída (Câmara et al., 2001).

A lógica Fuzzy é indicada sempre que se tiver que lidar com ambiguidades (Câmara et al., 2001). Há vários operadores para produzir um mapa síntese por lógica Fuzzy, dentre os quais o produto algébrico, a soma algébrica e o operador 
gamma. No operador produto algébrico o valor de pertinência Fuzzy de saída é a multiplicação dos valores dos pixels dos mapas de entrada referente a cada critério. Os valores de pertinência tendem a ser muito pequenos quando se utiliza este operador, devido ao efeito de se multiplicar diversos números menores do que 1 . A operação por soma algébrica não consiste em uma simples soma algébrica dos mapas de entrada, mas representa um operador complementar ao produto algébrico. Assim, o resultado é sempre um valor maior ou igual ao maior valor de pertinência fuzzy de entrada (Câmara et al., 2001).

O operador gamma é definido pela multiplicação de dois termos: uma soma algébrica e um produto algébrico, sendo ambos elevados a um expoente gamma.

\section{$\mu=(\text { Soma algébrica Fuzzy) })^{\gamma} \mathrm{x}$ (Produto algébrico Fuzzy) $)^{1-\gamma}$}

A importância de cada termo (soma ou produto algébrico Fuzzy) no operador gamma é definida atribuindo-se valores entre 0 e1 para o expoente " $\gamma$ ". Nesse trabalho, adotamos três variações para esse expoente, sendo os valores 0,4 ; 0,6 e 0,8 para identificar a melhor dispersão do conjunto de pontos de cada modelo.

\section{Validação do mapa de vulnerabilidade à erosão por algoritmo de aprendizagem de máquina}

Um total de 1902 pontos foram utilizados na validação do mapa de vulnerabilidade à erosão produzido para a bacia do rio Pirapozinho. Desses, 951 correspondem a locais de erosão na área em estudo, os quais foram determinados pela fotointerpretação de Imagens do Google Earth Pro.

Para gerar os pontos 951 sem erosão na bacia de interesse, inicialmente geramos aleatoriamente (ferramena Point Sampling Tool) esses pontos com o software QGIS $3.8 \mathrm{e}$, posteriormente, verificamos visualmente nas imagens do Google Earth Pro se algum desses pontos encontravam-se em áreas de erosão. Em caso afirmativo, esse ponto foi substituído por um ponto sem erosão. Posteriormente, aos pontos que correspondem a áreas de erosão, foi atribuído o valor 1 , e o valor 0 aos pontos sem erosão.

Para cada localização dos pontos de validação (representando locais com e sem erosão), foi extraído o valor de pixel correspondente em cada um dos modelos de inferência aplicados. Esses dados foram organizados em uma tabela e carregados no software Orange 3.21. Nesse ambiente de aprendizagem de máquina, adotou-se o algoritmo de regressão logística para determinar a acurácia de cada modelo de incerteza criado. Para tanto, foi selecionado o dado de referência (pontos de validação) como alvo (target) e os dados provenientes do modelo de inferência como característica (feature). Com isso, o modelo de regressão logística determinada (faz a predição) o valor do dado de referência com base no valor do dado de característica. A regressão logística foi aplicada com a regularização do tipo Ridge (L2) com força $\mathrm{C}=1$, considerando na validação da performance do algoritmo a validação cruzada em 10 k-folders em modo estratificado e taxa de aprendizagem de $0,01 \%$ em modo constante. Esses foram os valores que apresentaram maior estabilidade para o modelo de regressão diante do conjunto amostral utilizado. $\mathrm{O}$ processo de validação da acurácia do mapa de vulnerabilidade foi realizado individualmente para modelo de inferência utilizado.

A comparação do desempenho dos modelos de inferência foi realizada pelo calculado dos valores de AUC (Area Under the Curve Área sob a curva), Acurácia Global, F1-Score, Precisão e Sensibilidade. Além disso, para uma comparação visual do desempenho dos modelos, construiu-se a curva ROC (Receiver Operating Characteristic), para cada modelo de incerteza empregado. A ROC é um gráfico criado a partir dos valores de Taxa de Valores Positivos (TVP), também conhecido como sensibilidade do modelo, versus a Taxa de Valores Falso-Positivos para vários valores de limiares (threshold).

\section{Resultados e discussão}

Para cada modelo de inferência aplicado, obteve-se um mapa (Figura 2) que sintetiza a vulnerabilidade à erosão da bacia, a qual varia de baixa a alta vulnerabilidade. Em cada mapa, as regiões no matiz (cor) verde indicam menor vulnerabilidade à erosão, e quanto mais próximo ao vermelho, maior vulnerabilidade. Uma análise visual desses produtos revela que a variação no modelo lógico de inferência gera resultados muito distintos (comparação entre CLP e AHP com o modelo Fuzzy por operação de produto algébrico, por exemplo). Isso está relacionado à forma de construção do modelo de inferência, revelando a importância de se validar os resultados com verdades terrestres (pontos de verificação), para se garantir o nível de representatividade do mapa com a realidade em campo.

Outra análise visual dos resultados foi realizada pelo gráfico de dispersão do conjunto de pontos de validação, para cada modelo de inferência (Figura 3). Observamos que no modelo Fuzzy, com o operador gamma sendo o expoente 0,6 (Figura 3), os pontos com e sem erosão não se 
interceptam em nenhum momento. Isso mostra que esse modelo garantiu melhor separabilidade entre os pontos com erosão dos pontos sem erosão na área estudada. Uma análise descritiva desse modelo (Tabela 3) mostra que o valor mínimo dos pixels, representando pontos com erosão foi de 0,65 e o valor máximo para os pontos sem erosão foi de 0,21 , provando a capacidade desse operador em separar esses pontos com e sem erosão.

Os mapas gerados pelo modelo Fuzzy com o operador produto e gamma (com expoente de 0,4 ) foram muito similares (Figura 2). Enquanto o resultado com o gamma 0,6 e 0,8 são mais parecidos (Figura 2). Observamos que quanto maior o valor do expoente gamma, mais próximo o resultado foi do operador produto e quanto menor, mais próximo ao do operador soma.

O resultado gerado pelo modelo CLP é o que mais apresenta uma mistura entre os pontos com e sem erosão (Figura 2). Isso se confirma ao se analise a dispersão dos valores de mínimo e máximo dos pixels nesses pontos de validação (Figura 3).

O mapa síntese obtido pela modelagem Fuzzy por soma algébrica (Figura 2) mostra que a bacia em estudo se encontra com alto nível de vulnerabilidade à erosão em sua porção Noroeste. Esse mesmo resultado é ressaltado, porém, em menor intensidade, nos mapas sínteses gerados pelos demais modelos de inferência, com exceção ao modelo Fuzzy por produto algébrico. Assim como no trabalho de Fronza (2019), os operadores soma e produto algébrico apresentaram resultados muito restritivos ou muito permissivos quando aplicados.

A porção Noroeste da bacia do rio Pirapozinho apresenta alta densidade de drenagem associada com altos valores de declividade, e que esses critérios foram os que obtiveram maior peso na ponderação pelo AHP (Tabela 2).

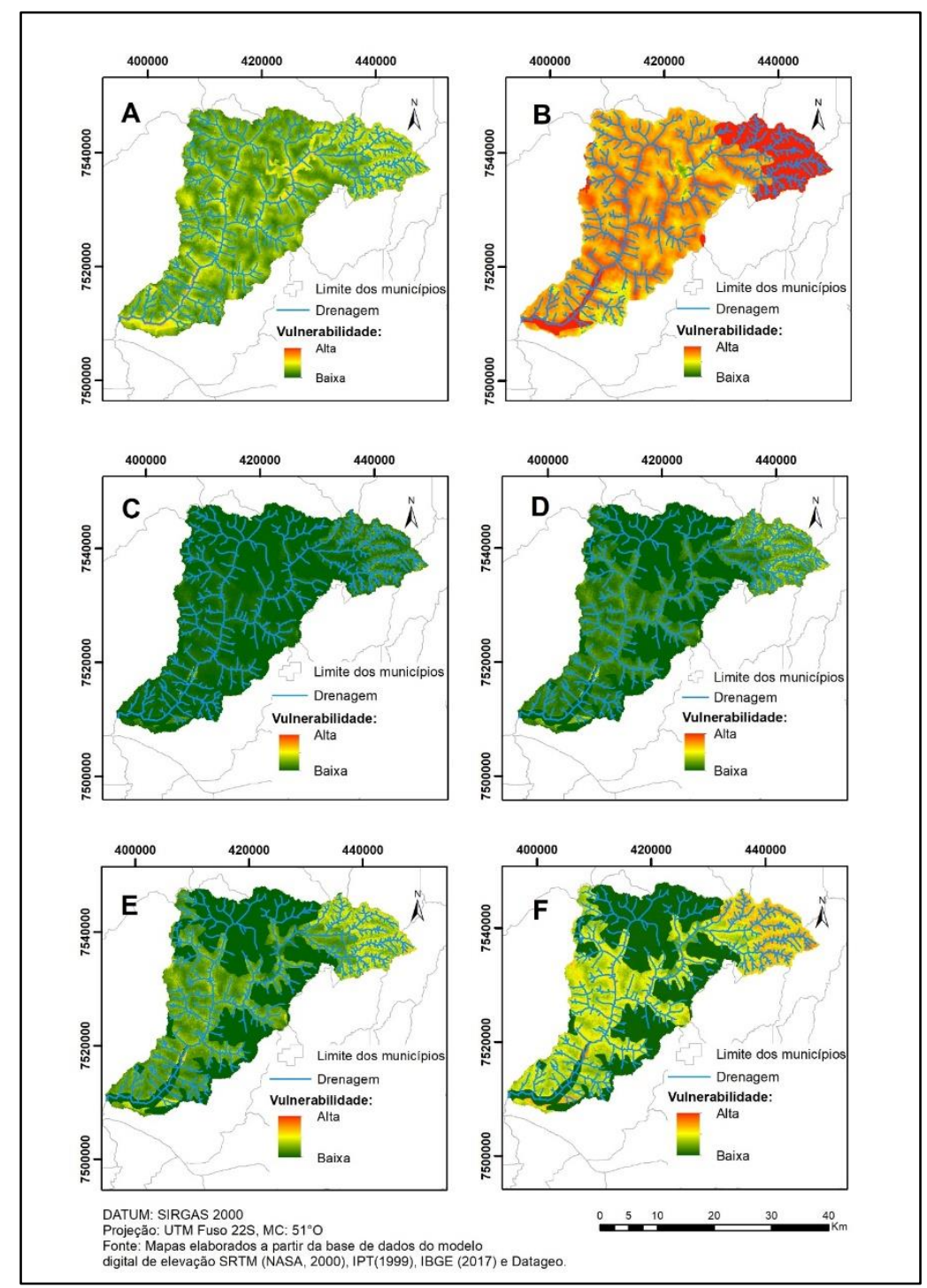

Figura 2. Mapa de vulnerabilidade à erosão para a bacia hidrográfica do rio Pirapozinho obtido por diferentes modelos de inferência geográfica. (A) AHP e CLP; (B) Fuzzy por soma algébrica; (C) Fuzzy por produto 
algébrico; (D)Fuzzy por operador gamma com expoente de 0,4; (E) Fuzzy por operador gamma com expoente de 0,6 e; (F) Fuzzy por operador gamma com expoente de 0,8 .
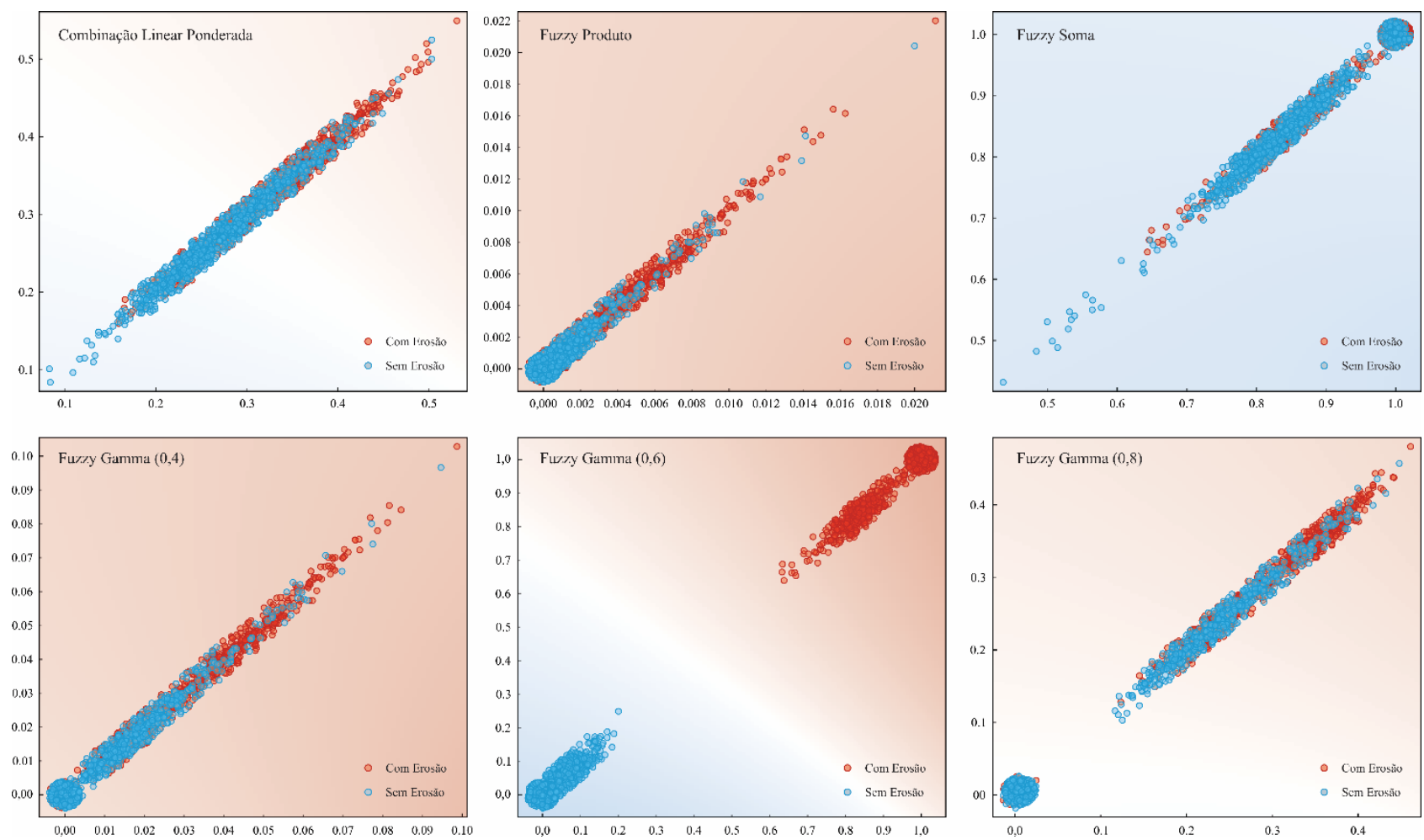

Figura 3. Dispersão do conjunto de pontos (com e sem erosão) para cada modelo de inferência usado.

Tabela 3. Valores descritivos de cada conjunto amostral.

\begin{tabular}{|c|c|c|c|c|c|c|c|c|c|c|c|c|}
\hline \multirow[t]{2}{*}{ Métrica } & \multicolumn{2}{|c|}{ CLP } & \multicolumn{2}{|c|}{ Fuzzy Produto } & \multicolumn{2}{|c|}{ Fuzzy Soma } & \multicolumn{2}{|c|}{$\begin{array}{c}\text { Fuzzy Gamma } \\
(0,4)\end{array}$} & \multicolumn{2}{|c|}{$\begin{array}{c}\text { Fuzzy Gamma } \\
(0,6)\end{array}$} & \multicolumn{2}{|c|}{$\begin{array}{c}\text { Fuzzy Gamma } \\
(0,8)\end{array}$} \\
\hline & 0 & 1 & 0 & 1 & 0 & 1 & 0 & 1 & 0 & 1 & 0 & 1 \\
\hline Valor mínimo & 0,08 & 0,15 & 0,00 & 0,00 & 0,43 & 0,65 & 0,00 & 0,00 & 0,00 & 0,65 & 0,00 & 0,00 \\
\hline Valor máximo & 0,50 & 0,53 & 0,02 & 0,02 & 1,00 & 1,00 & 0,09 & 0,09 & 0,21 & 1,00 & 0,45 & 0,46 \\
\hline Média & 0,27 & 0,32 & 0,00 & 0,00 & 0,85 & 0,92 & 0,01 & 0,03 & 0,03 & 0,92 & 0,14 & 0,24 \\
\hline Desvio-padrão & 0,06 & 0,06 & 0,00 & 0,00 & 0,09 & 0,08 & 0,01 & 0,02 & 0,01 & 0,09 & 0,13 & 0,12 \\
\hline Mediana & 0,27 & 0,32 & 0,00 & 0,00 & 0,84 & 1,00 & 0,00 & 0,02 & 0,00 & 1,00 & 0,16 & 0,26 \\
\hline $\begin{array}{l}\text { Coeficiente de } \\
\text { Variação }\end{array}$ & 22,63 & 18,97 & 173,17 & 98,40 & 10,61 & 9,47 & 124,43 & 71,19 & 108,20 & 9,47 & 93,88 & 49,66 \\
\hline
\end{tabular}

Sem erosão (0); com erosão (1). CLP = Combinação Linear Ponderada.

As curvas ROC mostradas na Figura 4 permitem comparar os modelos de inferência, além de demonstrar o desempenho individual máximo de cada um deles. Esse desempenho é medido a partir dos valores de AUC, o qual tem sido abordado em diversos trabalhos para diferentes aplicações (Iasevoli et al., 2018; Closas et al., 2018; Kuremoto et al., 2018). A inferência pelo modelo Fuzzy gamma com expoente de 0,6 apresentou a maior curva ROC e teve maior AUC, seguida do modelo gamma 0,8 e 0,4 , respectivamente (Tabela 4). O gamma 0,6 não apresentou nenhum ponto classificado erroneamente (ponto de erosão predito como ponto sem erosão, por exemplo), seja para as áreas com erosão, seja para as áreas sem erosão.
Por essa razão a sua acurácia, precisão e sensibilidade foram iguais a $100 \%$. Os demais modelos apresentaram precisão entre 65 e $69 \%$ (Tabela 4), com exceção da modelagem Fuzzy com produto algébrico que teve pior resultado, na ordem de $25 \%$ sua precisão. A Figura 5 indica os modelos e as retas que melhor conseguiram separar cada valor predito pela regressão logística. As retas foram formadas a partir do valor de limiar que atingiu a melhor performance na curva ROC. Torna-se novamente evidente a capacidade do modelo gamma, com expoente 0,6 , em separar os pontos com erosão dos pontos sem erosão na área mapeada. 

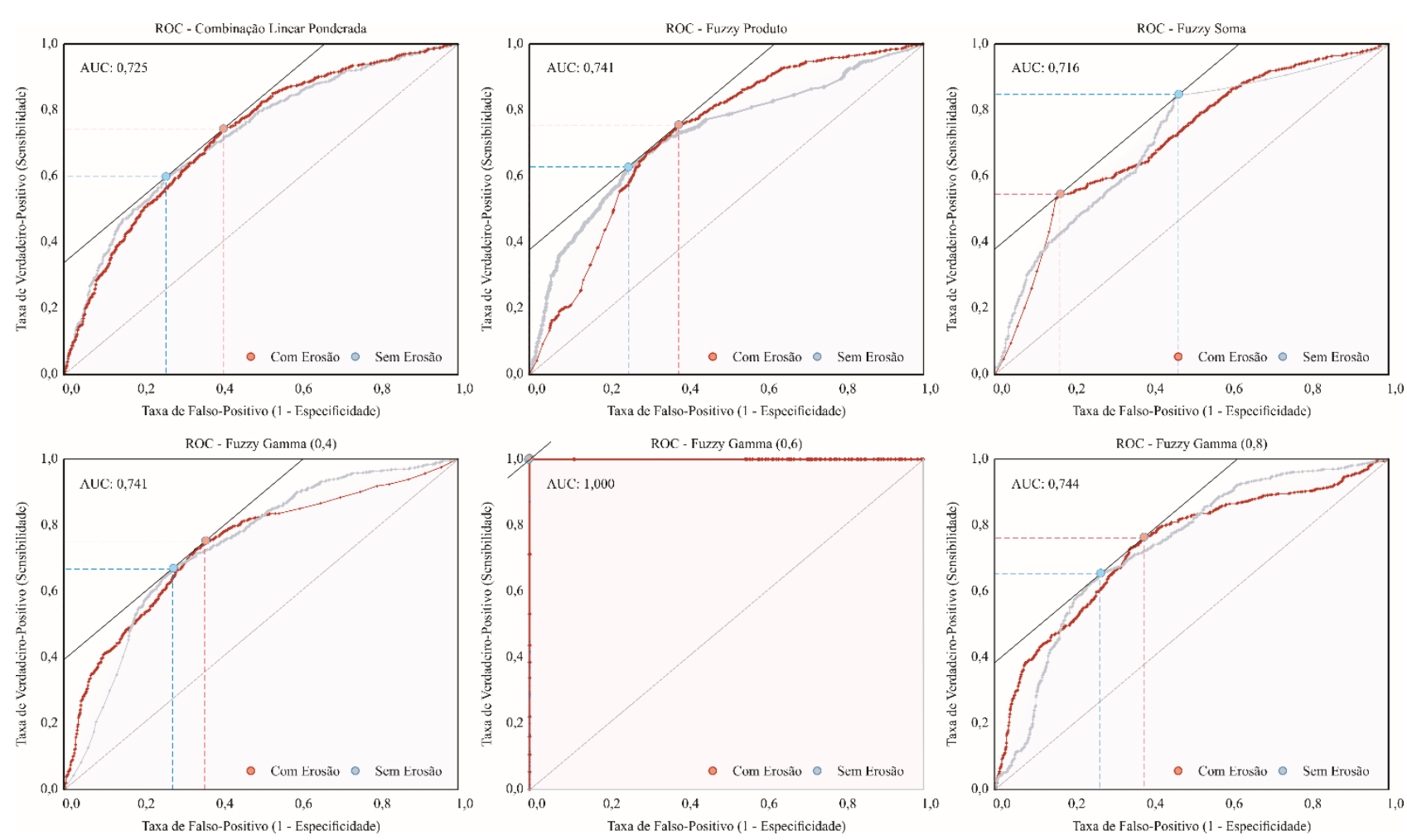

Figura 4. Curvas ROC da validação por regressão logística em cada modelo de incerteza.

Tabela 4 - Performance de cada modelo de incerteza de acordo com a regressão logística.

\begin{tabular}{cccccc}
\hline Método & AUC & Acurácia Global & F1-Score & Precisão & Sensibilidade \\
\hline CLP & 0,725 & $65,9 \%$ & $65,9 \%$ & $65,9 \%$ & $65,9 \%$ \\
Produto & 0,741 & $50,1 \%$ & $33,4 \%$ & $25,1 \%$ & $50,1 \%$ \\
Soma & 0,716 & $65,0 \%$ & $65,0 \%$ & $65,2 \%$ & $62,4 \%$ \\
Gamma $(0,4)$ & 0,741 & $67,9 \%$ & $67,8 \%$ & $68,1 \%$ & $67,9 \%$ \\
Gamma $(0,6)$ & 1,000 & $100,0 \%$ & $100,0 \%$ & $100,0 \%$ & $100,0 \%$ \\
Gamma $(0,8)$ & 0,744 & $68,2 \%$ & $67,8 \%$ & $69,3 \%$ & $68,2 \%$ \\
\hline \multicolumn{5}{c}{}
\end{tabular}

CLP $=$ Combinação Linear Ponderada.

Mostramos que nesse caso de vulnerabilidade à erosão o CLP não foi o método que apresentou a melhor acurácia, demonstrando então a importância da validação dos mapas. Os resultados confrontaram a literatura, uma vez que grande parte dos artigos (Rahman et al, 2009; Dias et al., 2014; Traficante et al., 2017; Vettorazzi et al., 2017; Rodrigues et al., 2018) usam a CLP e AHP em problemas de inferência, ou não abordam a acurácia dos resultados, como Ceconi et al. (2018), Richardson et al. (2018) e Ghoussein (2018). Diante disso, mostramos que mesmo o CLP sendo o método mais utilizado, nem sempre ele é o melhor. 

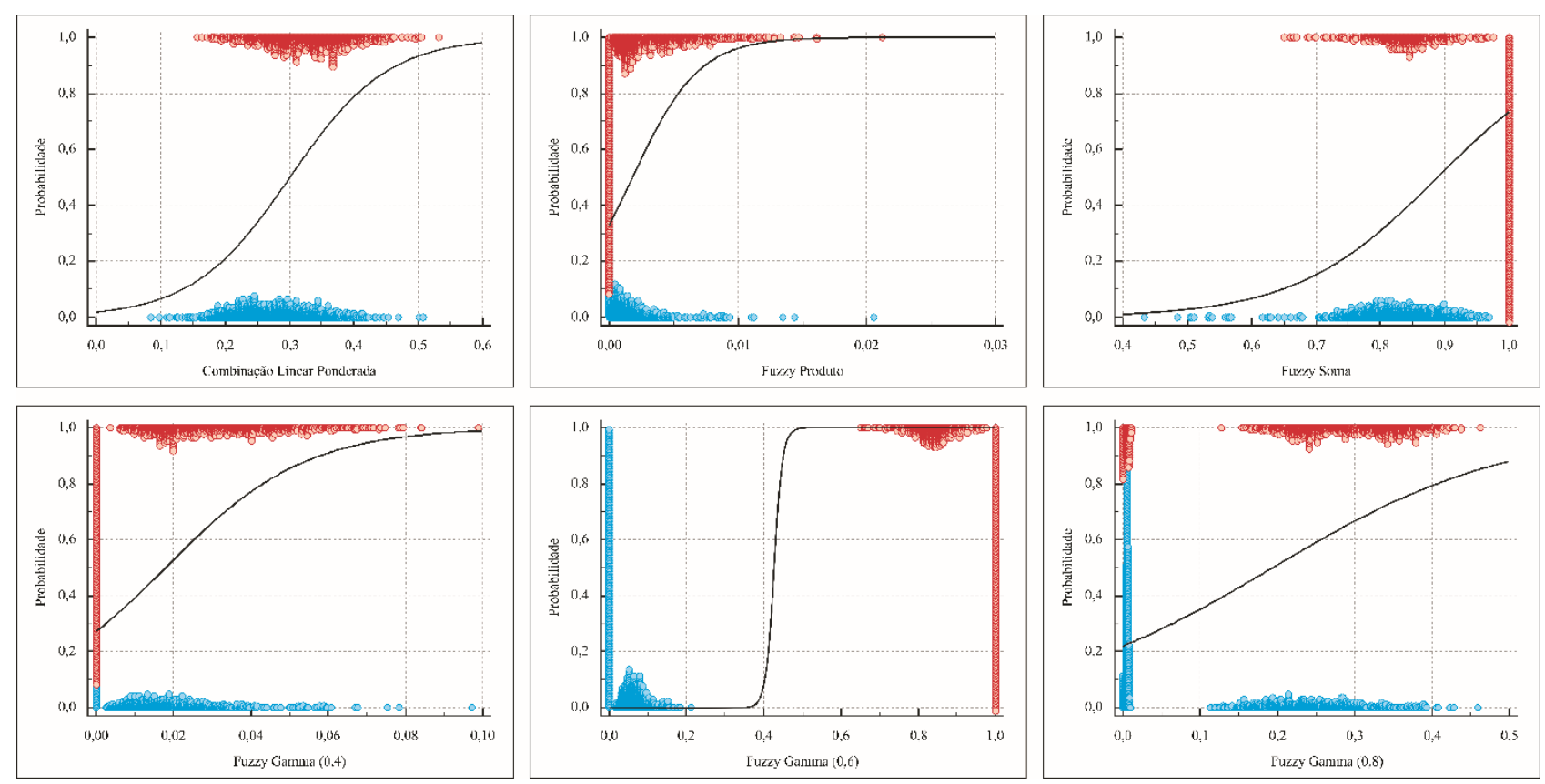

Figura 5. Gráfico dos pontos na regressão logística de acordo com o modelo empregado.

\section{Conclusão}

Conclui-se que o modelo de inferência Fuzzy com o operador gamma foi o que melhor se ajustou ao conjunto de dados de validação testados pelo algoritmo de regressão logística. Esse operador apresentou eficiência ótima quando o valor do expoente gamma 0,6 foi atribuído, mas quando adotado os valores 0,4 e 0,8 o comportamento não foi o mesmo e a performance foi reduzida. Isso nos permite concluir sobre a necessidade de testar diversos valores para esse parâmetro de expoente na modelagem Fuzzy pelo operador gamma.

A análise da acurácia por meio dos pontos de validação usando a repressão logística em ambiente de aprendizagem de máquina se mostrou uma abordagem eficaz. Isso porque permitiu identificar o mapa síntese que melhor representa a realidade em campo, ao se determinar nível de precisão dos modelos de inferência.

\section{Agradecimentos}

Agradecimento ao Núcleo de Estudos Ambientais e Geoprocessamento - NEAGEO e a Coordenação de Aperfeiçoamento de Pessoa de Nivel Superior (CAPES) pelo apoio financeiro mediante bolsa de mestrado PROSUP.

\section{Referências}

Bianchini, C.D.; Oliveira, G.G., 2019. Geoprocessamento aplicado à identificação de áreas aptas para a implantação de unidades de conservação no Vale do Taquari, RS. Revista Brasileira de Cartografia 71, 513-541.
Câmara, G.; Davis, C.; Monteiro, A.M.V., 2001. Introdução à Ciência da Geoinformação. INPE, São José dos Campos - SP.

Ceconi, D.E.; Piccilli, D.G.A.; Bernardi, F.; Fensterseifer, P., 2018. Análise de vulnerabilidade visando o planejamento ambiental em bacia de captação para abastecimento público. Revista Ambiente e Sociedade 21, 2-22.

Closas, A.H.; Franchini, N.B.; Kuc, L.C.; Dusicka, M.A.; Hisgen, C.M., 2018. Modelo logístico explicativo de las relaciones entre autoconcepto y rendimiento académico. Revista De La Facultad De Ciencias Económicas - UNNE 20, 187-208.

Dias, V.S.B.; Silva, A.B., 2014. AHP na Modelagem da vulnerabilidade ambiental do minicorredor ecológico Serra das Onças (BA). Revista Brasileira de Cartografia 66, 13631377.

Ding, S.; Zhang, Y.; Liu, B.; Kong, W.; Meng, W., 2013. Effects of riparian land use on water quality and fish communities in the headwater stream of the Taizi River in China. Frontiers of Environmental Science \& Engineering 7, p. 699-708.

Dobos, E.; Micheli, E.; Baumgardner, M.F.; Biehl, L.; Helt, T., 2000. Use of combined digital elevation model and satellite radiometric data for regional soil mapping. Geoderma 97, 367391.

Fronza, J.G., 2019. Lógica fuzzy na avaliação ambiental e alocação otimizada de traçados de linhas de transmissão. Simpósio Brasileiro de 
Sensoriamento Remoto. ISBN: 978-85-1700097-3.

Fushimi, M.; Nunes, J.O.R., 2018. Vulnerabilidade ambiental aos processos erosivos lineares das paisagens de parte dos municípios de Marabá Paulista (SP) e Presidente Epitácio (SP), Brasil. Revista da Associação Nacional de Pós-Graduação e Pesquisa em Geografia (Anpege) 14, 05-27.

Fushimi, M.; Nunes, J.O.R.; Nakamura, R.Y.; TAKATA, L.T.O., 2013. Vulnerabilidade ambiental e aplicação de técnicas de contenção aos processos erosivos lineares em áreas rurais no município de Presidente Prudente - SP. Revista Brasileira de Geomorfologia 14, 343-356.

Ghoussein, Y.; Mhawej, M.; Jaffal, A.; Fabel, A.; Hourany, R.E.; Faour., G., 2018. Vulnerability assessment of the SouthLebanese coast: A GIS-based approach. Ocean and Coastal Management 158, 56-63.

Gonçalves, S.R.A.; Araújo, R.R.; Imai, N.N., 2016. Mapeamento do grau de fragilidade com processo analítico hierárquico e operadores fuzzy gama na detecção áreas de fragilidade ambiental. Revista Brasileira de Cartografia 68, 327-337.

Iasevoli, F.; Avagliano, C.; Altavilla, B.; Ciccarelli, M.; D’Ambrosio, L.; Francesco, D. N.; Razzino, E.; Fornaro, M.; Bartolomeis, A., 2018. Evaluation of a few discrete clinical markers may predict categorization of actively symptomatic non-acute schizophrenia patients as treatment resistant or responders: A study by ROC curve analysis and multivariate analyses. Psychiatry Research 269, 481-493.

Jesuz, C.R.; Cabral, I.L.L., 2019. Análise da erosão laminar em áreas com uso agrícola e pastagem: bacia hidrográfica do rio Tenente Amaral - MT. Geosul 34, 254-279.

Kuremoro, T.; Baba, Y.; Obayashi, M.; Mabu, S.; Kobayashi, K., 2018. Enhancing EEG Signals Recognition Using ROC Curve. Journal of Robotics, Networking and Artificial Life 04, 293-286.

Malczewski, J., 2010 Multiple Criteria Decision Analysis and Geographic Information Systems. In: GRECO, S.; EHRGOTT, M.; Figueira, J. R. Multiple Criteria Decision Analysis, 02, 369-395.

Mello, K.; Costa, D.R.; Valente, R.A.; Vettorazzi, C.A., 2018. Multicriteria Evaluation for Protected Area Definition Aiming at Water Quality Improvement. Floresta e Ambiente 25. doi: 10.1590/2179-8087.013416

Meneses, B.M.; Reis, R.; Vale, M.J.; Saraiva, R., 2015. Land use and land cover changes in
Zêzere watershed (Portugal) - Water quality implications. Science of the Total Environment, 527, 439-447.

Mota, M.T.; Bressane, A.; Roveda, J.A.F.; Roveda, S.R.M.M., 2019. Classification of successional stages in atlantic forests: a methodological approach based on a fuzzy expert system. Ciência Florestal 29, 519-530.

Nascimento, N.S.; Vieira, E.M.; Gonçalves, J.A.C.; Cunha, G.P.Q., 2016. Estudo da vulnerabilidade ambiental em uma micro bacia hidrográfica empregando hierarquia nominal e operador local. Revista Brasileira de Geografia Física 09, 897-916.

Nossack, F.A.; Zimback, C.R.L.; Silva, R.F.B.; Sartori, A.A.C., 2016. Aplicação de análise multicriterial para determinação de áreas prioritárias à recomposição florestal. Irriga Brazilian Jounal of irrigation and drainage, 19, 612-625.

Oliveira, P.T.S.; Rodrigues, D.B.B.; Alves Sobrinho, T.; Panachuki, E., 2009. Processo analítico hierárquico aplicado a vulnerabilidade natural a erosão. Geociência, São Paulo 28, 417-424.

Porto, D.T.; Basso, L.A.; Strohaecker, T.M., 2019. Diagnóstico ambiental da bacia hidrográfica do rio Mampituba, região Sul do Brasil, utilizando a Matriz FPEIR. Geosul, 34, 58-50.

Rahman, M.R.; Chongfa, C., 2009. Soil erosion hazard evaluation-An integrated use of remote sensing, GIS and statistical approaches with biophysical parameters towards management strategies. Ecological Modelling, 220, 17241734.

Ramos, A.P.M.; Rodrigues, B.M.; Osco, L.P.; Antunes, P.A., 2018. Abordagem sistemática de projeto cartográfico para a análise da qualidade ambiental de bacia hidrográfica. Revista Brasileira de Geografia Física, 11, 1079-1100.

Reis, T.E.; Costa, V.C., 2017. Análise da vulnerabilidade na zona de amortecimento do Parque Estadual do Ibitipoca -(MG), com o uso de SIG. Geosul, 32, 77-96.

Ribeiro, A.S.; Mincato, R.L.; Curi, N.; Kawakubo, F.S., 2016. Vulnerabilidade ambiental à erosão hídrica em uma sub-bacia hidrográfica pelo processo analítico hierárquico. Revista Brasileira de Geografia Física 09, 016-031.

Richardson, C.P.; Amankwatia, K., 2018. GISBased Analytic Hierarchy Process Approach to Watershed Vulnerability in Bernalillo County, New Mexico. Journal of Hydrologic Engineering 23.

Rodrigues, T.; Tommaselli, J.T.G.; Arroio Junior, P.P., 2018. Mapeamento da vulnerabilidade 
aos processos erosivos a partir de métodos multicriteriais: um estudo sobre as condições ambientais da bacia hidrográfica da represa da Laranja Doce, Martinópolis - SP. Revista Formação (Online), v. 25, n. 45, 67-91.

Ross, J.L.S., 1994. Análise empírica da fragilidade dos ambientes naturais e antropizados. Revista do departamento de Geografia, 08, 3-74.

Saaty, Thomas L., 1990. How to make a decision: the analytic hierarchy process. European Journal of Operational Research, NorthHolland. 48, 9-26.

Samizava, T.M.; Nunes, J.O.R.; Imai, N.N.; Kaida, R.H., 2008. Suavização dos contatos entre compartimentos de relevo através de modelagem por inferência Fuzzy: mapeamento geomorfológico no município de Presidente Prudente - SP - Brasil. Revista Brasileira de Geomorfologia, 09, 65-73.

Shi, P.; Zhang, Y.; Li, Z.; Li, P.; Xu, G., 2017. Influence of land use and land cover patterns on seasonal water quality at multi-spacial scales. Catena, v. 151, 182-190.

Silva, G.R.V.; Souza, Z.M.; Filho, M.V.M.; Barbosa, R.S.; Souza, G.S., 2012. Soil, water and nutrient losses by interrill erosion from green cane cultivation. Revista Brasileira de Ciência do Solo 36, 963-970.

Silva, P.L.; Andrade, L.N.P.S.; Leandro, G.R.S.; Souza, A.S., 2017. Evolução dos processos erosivos e aspectos granulométricos em um sistema de voçoroca no Distrito de Vila Aparecida, município de Cáceres - Mato Grosso. Revista Brasileira de Geografia Física 10, 627-640.

Silva, V.C.B.; Machado, P.S., 2014. SIG na análise ambiental: susceptibilidade erosiva da bacia hidrográfica do córrego Mutuca, Nova Lima Minas Gerais. Revista de Geografia (UFPE) 31, 66-87.
Traficante, D.P.; Campos, S.; Manzione, R.L.; RODRIGUES, B.T., 2017. Fragilidade ambiental da bacia hidrográfica do rio Capivara, Botucatu-SP. Energia na agricultura 32, 88-95.

Trevisan, D.P.; Moschini, L.E.; Dias, L.C.C.; Gonçales, J.C., 2018. Avaliação da vulnerabilidade ambiental de São Carlos - SP. O espaço geográfico em análise 44, 272-288.

Valente, R.O.A.; Vettorazzi, C.A., 2005. Comparação entre métodos de avaliação multicriterial, em ambiente SIG, para a conservação e a preservação florestal. Scientia Forestalis 26, 51-61.

Valente, R.O.A.; Vettorazzi, C.A., 2008. Definition of priority areas for forest conservation through the ordered weighted averaging method. Forest Ecology and Management 256, 1408-1417.

Valente, R.O.A.; Vettorazzi, C.A., 2016. Priority areas for forest restoration aiming at the conservation of water resources. Ecological Engineering 94, 255-267.

Valente, R.A.; Petean, F.C.S.; Vettorazzi, C.A., 2017. Multicriteria decision analysis for prioritizing areas for forest restoration. CERNE 23, 53-60.

Viel, J.A.; Rosa, K.K.; Hoff., 2017. Estudo da erosão superficial do solo por meio de SIG na região da denominação de origem Vale dos Vinhedos (Brasil). Revista Brasileira de Geomorfologia 18.

Voogd, J., 1983. Multicriteria evaluation for urban and regional planning. London: Pio, 125.

Yang, H.; Wang, G.; Wang, L.; Zheng, B., 2016. Impact of land use change on water quality in headwaters of the Three Gorges Reservoir. Environmental Science and Pollution Research 23, 11448-11460. 